\title{
Une analyse par quantiles de la résilience chez les élèves issus de milieux défavorisés
}

\section{A quantile analysis of resilience among students from economically disadvantaged backgrounds \\ Un análisis en cuantiles de la resiliencia entre alumnos provenientes de medios desfavorecidos}

\section{Gabriel Power et Lucie DeBlois}

Volume 39, numéro 1, printemps 2011

Regards critiques sur les discours politiques et scientifiques à l'égard de la réussite scolaire

URI : https://id.erudit.org/iderudit/1004332ar

DOI : https://doi.org/10.7202/1004332ar

Aller au sommaire du numéro

Éditeur(s)

Association canadienne d'éducation de langue française

ISSN

0849-1089 (imprimé)

1916-8659 (numérique)

Découvrir la revue

Citer cet article

Power, G. \& DeBlois, L. (2011). Une analyse par quantiles de la résilience chez les élèves issus de milieux défavorisés. Éducation et francophonie, 39(1), 93-118. https://doi.org/10.7202/1004332ar
Résumé de l'article

Une analyse prenant appui sur la notion de capital social et de résilience a permis d'élaborer un modèle de création du capital humain de l'élève. En utilisant ce modèle, nous avons voulu cerner les facteurs qui pourraient favoriser la réussite scolaire des élèves de 15 ans issus de milieux défavorisés, réussite interprétée comme un développement de leur capital humain. Nous avons étudié les résultats aux questionnaires de l'élève de Pisa 2000 et nous les avons interprétés en fonction des dimensions structurales, normatives et interactives qui sont générées par le capital social de l'école et de la famille et par la résilience de l'élève. Nous observons des corrélations positives pour des variables en relation avec les dimensions structurales et interactives. Toutes les variables en relation avec la dimension normative révèlent des corrélations négatives. Nous présentons ces résultats et précisons des hypothèses pour expliquer ces résultats. Enfin, nous dégageons des critères permettant de définir la réussite scolaire comme un capital scolaire.
Tous droits réservés (C) Association canadienne d'éducation de langue française, 2011 cest protége par la loi sur le droit d'auteur. L'utilisation des services d'Érudit (y compris la reproduction) est assujettie à sa politique d'utilisation que vous pouvez consulter en ligne. 


\title{
Une analyse par quantiles de la résilience chez les élèves issus de milieux défavorisés ${ }^{1}$
}

\author{
Gabriel POWER \\ Université Laval, Québec, Canada \\ Lucie DEBLOIS ${ }^{2}$ \\ Université Laval, Québec, Canada
}

\section{RÉSUMÉ}

Une analyse prenant appui sur la notion de capital social et de résilience a permis d'élaborer un modèle de création du capital humain de l'élève. En utilisant ce modèle, nous avons voulu cerner les facteurs qui pourraient favoriser la réussite scolaire des élèves de 15 ans issus de milieux défavorisés, réussite interprétée comme un développement de leur capital humain. Nous avons étudié les résultats aux questionnaires de l'élève de Pisa 2000 et nous les avons interprétés en fonction des dimensions structurales, normatives et interactives qui sont générées par le capital social de l'école et de la famille et par la résilience de l'élève. Nous observons des corrélations positives pour des variables en relation avec les dimensions structurales et interactives. Toutes les variables en relation avec la dimension normative révèlent des corrélations négatives. Nous présentons ces résultats et précisons des hypothèses pour expliquer ces résultats. Enfin, nous dégageons des critères permettant de définir la réussite scolaire comme un capital scolaire.

1. Cette recherche a été possible grâce au financement du programme Initiative de recherche en éducation du CSCE-CRSH, numéro 539-2003-5.

2. Je tiens à remercier madame Marie-Pierre Dencuff qui a contribué à la rédaction de cet article par nos discussions. 


\section{ABSTRACT}

\section{A quantile analysis of resilience among students from economically disadvantaged backgrounds}

Gabriel POWER

Laval University, Québec, Canada

Lucie DEBLOIS

Laval University, Québec, Canada

An analysis based on the concept of social capital and resilience led to a model for the creation of student human capital. Using this model, we wanted to identify factors that could promote school success for 15 year-old students from economically disadvantaged backgrounds, success interpreted as development of their human capital. We studied the results of the Pisa 2000 questionnaires that we gave to the students, and interpreted them based on structural, normative and interactive dimensions generated by the social capital of school and family, and by the student's resilience. We observe positive correlations for structural and interactive variables. All of the normative variables reveal negative correlations. We present these results and elaborate hypotheses to explain them. Finally, we determined the criteria for defining school success as scholastic capital.

\section{RESUMEN}

\section{Un análisis en cuantiles de la resiliencia entre alumnos provenientes de medios desfavorecidos.}

Gabriel POWER

Universidad Laval, Quebec, Canadá

Lucie DEBLOIS

Universidad Laval, Quebec, Canadá

Un análisis fundado en las nociones de capital humano y de resiliencia permitió elaborar un modelo de creación de capital humano del alumno. Utilizando ese modelo, tratamos de circunscribir los factores que pueden favorecer el éxito escolar de alumnos de 15 años provenientes de medios desfavorecidos, éxito interpretado como desarrollo de su capital humano. Hemos estudiado los resultados de los cuestionarios del alumno de Pisa 2000 y lo hemos interpretado en función de las dimensiones estructurales, normativas e interactivas generadas por el capital social de la escuela, la familia y la resiliencia del alumno. Observamos correlaciones positivas para las variables relacionadas con las dimensiones estructurales e interactivas. Todas las variables relacionadas con la dimensión normativa muestran correlaciones negativas. Presentamos dichos resultados y precisamos hipótesis explicativas. Finalmente, despejamos algunos criterios que permiten definir el éxito escolar en tanto que capital escolar. 
It is a rare public policy initiative that promotes fairness and social justice and, at the same time, promotes productivity in the economy and in society at large. Investing in disadvantaged young children is such a policy. - James J. Heckman, lauréat du prix Nobel pour la science économique, billet paru dans le Wall Street Journal du 10 janvier 2006.

\section{Introduction}

Co-lauréat du prix Nobel de chimie en 2007, l'Italien Mario Capecchi a pourtant vécu dans la rue et sans ses parents entre l'âge de quatre et huit ans. Gamin des rues durant les années de la Deuxième Guerre mondiale, il retrouve sa mère après l'armistice et les deux sont invités par son oncle à aller vivre avec lui en Pennsylvanie aux États-Unis (Lee, 2007). Son parcours l'emmène à l'Université Harvard où il obtient un doctorat en biophysique et entame une carrière en biologie aux côtés de James Watson, lui-même lauréat d'un Nobel pour la découverte de l'ADN. Les traits caractéristiques qui ont permis à Capecchi de traverser une enfance douloureuse pour devenir un chercheur de renommée mondiale sont bien entendu difficiles à identifier, mais ils peuvent être considérés comme une forme de résilience.

$\mathrm{Au}$ cœur de la présente étude se trouvent les concepts de capital social et de résilience chez l'enfant. Une analyse conceptuelle déjà réalisée (Zhang et al. 2008) a permis d'élaborer un modèle qui vise à interpréter des résultats empiriques. Il sera dès lors possible de dégager des questions à propos de la réussite scolaire afin d'offrir une définition de la réussite scolaire.

\section{Problématique}

Au Canada, un nombre de plus en plus grand d'enfants sont touchés par des facteurs qui mettent en danger leur transition au monde adulte (Schonert-Reichl, 2000). Selon Willms (2002), entre $14 \%$ et $16 \%$ des enfants canadiens sont à risque sur le plan du développement cognitif et entre $17 \%$ et $22 \%$ le sont sur le plan du comportement. Dans ces conditions, la promotion d'une éducation de qualité et adaptée aux besoins des élèves demeure un défi. Dans leur présentation à la National Academy of Sciences américaine, Knudsen et al. (2006, p. 10155) expliquent qu'investir dans l'éducation des enfants issus de milieux défavorisés est de grande importance, parce que le développement cognitif qui devrait avoir lieu en bas âge est fort difficile à générer à un âge plus avancé.

Ainsi, pour étudier le problème des élèves à risque d'échec scolaire et de transition, il est possible de chercher à cerner les facteurs de risque, tels que la violence, la pauvreté, la discorde familiale, la violence envers les enfants et les abus de substances, et les difficultés des parents pour les éliminer ou les réduire (Masten et Coatsworth, 1998; Willms, 2002). Nous cherchons plutôt à encourager et renforcer certaines qualités et certains facteurs contextuels pour susciter la réussite scolaire et 
favoriser une adaptation sociale et professionnelle harmonieuse. Par exemple, une surveillance parentale structurée, des buts ambitieux, la sécurité à l'école pourraient être des facteurs à considérer. La présente étude cherche à documenter les facteurs qui aident les élèves à risque à surmonter l'adversité.

\section{Cadre théorique: un modèle à l'épreuve}

D'une part, la recherche en éducation portant sur le capital social se concentre surtout sur l'accumulation de capital social et économique comme déterminants du capital humain. Selon la théorie du capital social, le capital humain de l'enfant se structure et se développe grâce au capital humain et financier de la famille, de la communauté et de l'école. Pour l'Organisation de coopération et de développement économiques (OCDE), le capital social est un «filet social» ayant des normes et des valeurs qui favorisent la coopération (Cote et Healy, 2001). Pour Putnam (1995), le capital social rend possible la poursuite d'objectifs communs par les membres d'une communauté. Dans le contexte de l'éducation, la notion de capital social doit être conceptualisée en fonction du développement du capital humain de l'élève.

D'autre part, même s'il n'existe pas de définition de la résilience qui soit univoque, certains éléments sont à la base de cette notion: une adaptation réussie en présence de l'adversité. Les recherches portant sur la résilience montrent ainsi que certains facteurs peuvent aider les enfants issus de milieux socioéconomiquement défavorisés à surmonter à la fois les obstacles et les conséquences négatives de leur environnement (Maste, 1994; Nettles et Pleck, 1993; Rutter, 1987). Ces études mettent en lumière le rôle important que peut jouer l'école (Bernard, 1991; Bertrand, 2002; Coleman, 1988; Raudenbush et Willms, 1991; Rothman, 1995; Zhang, 2002). Dans ces conditions, lorsque ces formes de capital sont plus faibles (par exemple pour les enfants issus de milieux défavorisés), le rôle de l'école pourrait en effet devenir déterminant.

Zhang, DeBlois, Kamanzi, Deniger (2008) ont élaboré un modèle théorique à la suite d'une analyse conceptuelle des notions de capital social et de résilience. Les auteurs de ce modèle ont voulu articuler entre eux les concepts de capital social et de résilience. Ainsi conceptualisé, le capital humain de l'élève se situe dans une structure où les relations entre l'enfant et ses parents, mais aussi avec les autres adultes de la communauté et ses pairs, prennent une grande importance. 
Schéma 1. Modèle de création, à travers le capital social, d'un capital humain chez les élèves issus de milieux défavorisés

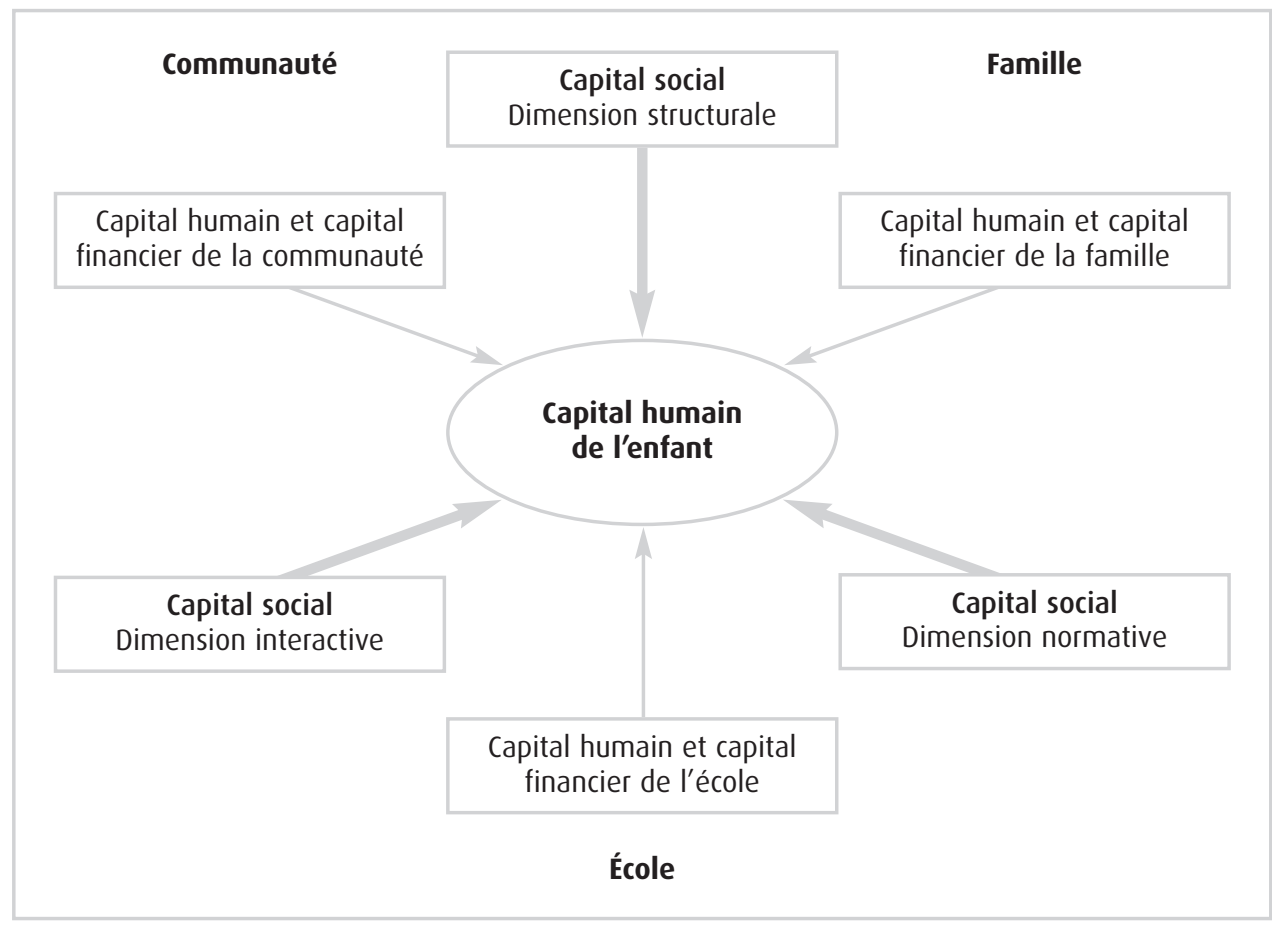

Ce modèle situe l'enfant au centre de trois environnements immédiats: la famille, la communauté et l'école. Dans ce modèle, le capital humain d'un enfant se compose du résultat des interactions du capital humain et du capital financier de la communauté, de la famille et de l'école, orchestré par le capital social. Dans ces conditions, le capital social se manifeste par trois dimensions: la dimension structurale, la dimension normative et la dimension interactive.

La première se définit par une présence parentale à domicile, la disponibilité d'activités parascolaires et communautaires destinées expressément aux élèves, la présence de réseaux et d'organisations d'élèves. Quant à la dimension normative, elle se présente par la discipline imposée à domicile et à l'école, par les attentes élevées de la part des parents, des enseignants et des amis. Enfin, la dimension interactive se manifeste par les interactions entre parent et enfant dans les discussions et l'aide aux devoirs, les interactions entre enseignant et enfant dans les échanges de questions et de réponses en classe et les interactions entre les pairs lors des formules d'apprentissage coopératif et de projets en groupe, la participation à des activités communautaires (service à la communauté, bénévolat local) et culturelles (visites au théâtre, concerts et autres activités culturelles). Nous intégrons aussi à la dimension interactive les interactions entre les élèves ainsi que les contextes et les situations d'apprentissage. 
Le capital humain de l'enfant se manifeste ainsi par l'expression de ses habiletés sociales et par ses compétences. Ce modèle a été mis à l'épreuve pour interpréter certaines analyses. Ainsi, l'utilisation des questionnaires adressés aux élèves et aux enseignants réalisés par Pisa 2000 et 2003 a permis d'interpréter certains résultats portant sur la famille et sur l'école.

En ce qui concerne la famille, il a été possible de préciser, par exemple, qu'au Canada la scolarité du père, les possessions culturelles et le niveau de biens à la maison ont un effet modérateur (indirect) sur le niveau socioéconomique de la famille (DeBlois, Rousseau, Zhu, 2006). De plus, la communication culturelle telle qu'elle a été définie précédemment semble être le mode d'accompagnement parental le plus important pour susciter la réussite scolaire des adolescents interrogés (DeBlois, Rousseau, Deslandes, Nadeau, 2009). En outre, l'effet de la communication culturelle et de la communication sociale pourrait être en partie expliqué par le niveau socioéconomique familial. Enfin, Rousseau, DeBlois et Lapointe (2008) ont observé que les différences entre les filles et les garçons variaient selon les contextes sociaux et culturels.

En ce qui concerne l'école, les résultats de Kamanzy, DeBlois, Deniger et Zhang (2006) montrent que, quelle que soit leur origine socioéconomique, les élèves qui obtiennent un résultat supérieur à la moyenne canadienne sont ceux qui bénéficient de l'apport de la variable ressources éducatives à l'école (livres, ordinateurs, etc.). En outre, c'est à la dimension interactive que revient la plus forte corrélation avec la réussite des élèves (DeBlois, Rousseau, et Zhu, 2006), ce qui confirme l'importance des interactions déjà observées pour la composante familiale. Précisons enfin que nos analyses révèlent aussi que le climat de l'école est sous l'influence de sa composition socioéconomique (DeBlois, Lapointe et Rousseau, 2007). Ainsi, plus la composition socioéconomique des élèves de l'école est diversifiée, plus le climat scolaire pourra favoriser la réussite scolaire. Ces résultats conduisent à reconnaître l'importance de porter une attention aux conditions des personnes qui soutiennent les élèves issus de milieux socioéconomiques défavorisés.

La présente étude vise à préciser les rôles des différentes dimensions du capital social dans la création de capital humain chez l'enfant afin d'évaluer à quel point les différentes variables associées soit au capital social ou au capital humain et financier de l'école sont pertinentes pour aider les élèves issus de milieux socioéconomiques défavorisés. L'hypothèse mise à l'épreuve soutient que l'école peut et doit compenser le plus faible niveau de capital humain chez les élèves issus de milieux défavorisés. Partant de cette hypothèse, les questions suivantes sont posées:

- Jusqu'à quel point le capital social et le capital humain et financier de l'école peuvent-ils appuyer les élèves de milieux défavorisés et compenser les effets d'un milieu familial socioéconomique défavorisé?

- Quelles composantes du capital scolaire (humain et financier) présentent les plus grandes corrélations sur le capital humain de l'élève?

- Dans ce contexte, comment définir la réussite scolaire? 


\section{La méthodologie}

\section{Description de la source de données: I'enquête PISA pour le Canada}

Les données proviennent des résultats pour le Canada de l'enquête du Programme international pour le suivi des acquis des élèves (PISA) de l'année 2000. Lâge moyen des élèves évalués est 15 ans 9 mois et les examens portent essentiellement sur la compréhension de l'écrit et, dans une moindre mesure, sur les mathématiques et la culture scientifique.

Pour les besoins de cette étude, nous avons défini un élève comme étant issu d'un milieu défavorisé si une de deux conditions s'applique: 1) l'élève vit dans une famille dont le niveau socioéconomique est peu élevé ou 2) l'élève a des difficultés d'apprentissage ou des troubles de comportement. Enfin, un élève est défini comme doublement défavorisé si les deux conditions s'appliquent.

Cette enquête est choisie sur la base de la fidélité de l'information contenue sur la performance scolaire des élèves canadiens. En plus des valeurs plausibles enre gistrées pour les résultats aux examens en mathématiques, science et lecture, l'enquête contient également un grand nombre de variables utiles se rapportant aux caractéristiques des écoles, leurs administrateurs et leur personnel enseignant.

Aux fins de la présente analyse, la variable dépendante qui sert de mesure pour la réussite scolaire est la valeur numérique (définie comme "valeur plausible») du test de littératie PISA 2000 au Canada. La littératie est définie comme étant la «capacité de comprendre, d'utiliser et d'analyser des textes écrits afin de pouvoir réaliser des objectifs personnels, développer des connaissances et des capacités et prendre une part active dans la société» (Statistique Canada, 2008). La valeur plausible telle que construite par l'enquête PISA est définie comme étant une mesure sans biais (au sens statistique) de la performance des élèves aux tests. Toutefois, il ne s'agit pas précisément du résultat de l'élève au test lui-même (pour plus de détails techniques, voir OCDE, 2005). L'analyse tient compte du sexe et du niveau socioéconomique enregistré dans la base de données. Pour établir l'échantillon des élèves de milieux défavorisés, nous utilisons l'index socioéconomique généré par l'étude PISA, qui se base sur les professions du père et/ou de la mère, du niveau d'éducation le plus élevé chez ces derniers et des variables qui décrivent le bien-être financier du foyer. Selon l'index, une étudiante ou un étudiant dans le premier quartile de la distribution est classé comme socioéconomiquement désavantagé, sinon il est classé comme socioéconomiquement favorisé. En particulier, cela nous permet d'évaluer l'hypothèse qu'il existe des différences entre la composition des variables significatives chez les élèves de milieux défavorisés qui réussissent et cette composition chez ceux qui réussissent moins bien. À moins d'indication contraire, tous les tests d'hypothèse sont symétriques et utilisent le seuil de $5 \%$ pour le choix de la valeur critique du test. Pour qu'une variable possède un effet significatif, nous exigeons ainsi au minimum qu'un test de Student rejette l'hypothèse nulle d'un effet égal à zéro selon le seuil de $5 \%$ et aussi que la valeur du coefficient lui-même soit non négligeable.

L'échantillon utilisé est défini de la façon suivante. Nous conservons d'abord toutes les observations pour le Canada. Les variables indépendantes non utilisées 
pour le Canada sont éliminées. Pour éviter de surcharger le modèle, seulement les variables indépendantes justifiées par la théorie sur la résilience de Zhang et al. (2008) dans le cadre scolaire sont conservées aux fins d'analyse empirique. Certaines variables d'identification sont conservées, comme le sexe et l'école, afin d'associer les données de l'élève avec les données de l'école provenant de la base de données PISA pour les écoles canadiennes.

Le modèle à estimer est le suivant: la variable dépendante est la valeur plausible au test de lecture dans l'enquête PISA 2000. Afin d'assurer l'interprétation des résultats avec ceux obtenus par d'autres chercheurs, nous utilisons les variables indices définies par PISA. L'utilisation des variables indices de PISA comporte l'avantage d'économiser en degrés de liberté, car chaque variable indice agit au lieu de plusieurs variables souvent dichotomiques (oui ou non). Enfin, ce choix réduit le nombre d'observations à éliminer pour cause de réponse manquante.

Les observations dans la base de données de PISA 2000 comportent des réponses manquantes, ce qui est habituel pour de telles enquêtes. Selon le statisticien Little (1992), une solution pour minimiser le biais causé par ces réponses manquantes est d'opter pour le "cas complet" (complete case), ce qui implique qu'on élimine toutes les observations qui contiennent des réponses manquantes en ne conservant que les observations «complètes». Évidemment, cette solution exige au départ un échantillon de grande taille afin que l'échantillon final demeure d'une taille convenable pour l'analyse. Pour notre analyse, cela nous laisse avec un nombre d'observations égal à 10303 . Pour de grands échantillons, cette solution est préférable à diverses stratégies d'interpolation qui cherchent à recréer les valeurs manquantes. De plus, si l'enquête touchait à des questions délicates, personnelles ou controversées, nous pourrions croire que l'absence de réponse pourrait être non aléatoire, car certains types de répondants hésiteraient davantage à répondre; autrement dit, le biais serait important. Mais rien dans la littérature ne suggère que tel est le cas ici. Tout de même, nous évaluons le biais possible occasionné par la stratégie «cas complet» en comparant la moyenne de la valeur plausible (résultat sur le test de lecture) dans l'échantillon original avec la moyenne dans l'échantillon "cas complet». Nous trouvons que le "cas complet» introduit un biais à la hausse faible et non significatif. Nous concluons alors que la stratégie du «cas complet» est correcte pour cette étude.

L'enquête PISA produit cinq estimations différentes de la valeur plausible au test de lecture. Selon PISA, les cinq estimations sont sans biais. Nous pouvons donc utiliser n'importe laquelle aux fins de cette étude (PISA, 2000). Par contre, pour obtenir une erreur-type correcte, ce qui est nécessaire pour déterminer quelles variables explicatives ont une association ou un effet positifs, il faut calculer une erreurtype selon la correction de Fay en utilisant les cinq estimations (PISA, 2000). Nous suivons cette approche pour chaque étape de l'analyse par quantiles.

\section{Définition et mesure des variables explicatives}

Il est utile de présenter, à titre descriptif, le contenu de certaines variables explicatives telles que construites et mesurées dans l'enquête PISA. 
- Pour une question portant sur le niveau de bien-être matériel à la maison, l'enquête PISA demande de combien de téléphones (cellulaires), téléviseurs, ordinateurs, automobiles et salles de bain dispose le foyer.

- Pour définir la variable «communication culturelle», le questionnaire de l'élève de PISA 2000 demande aux élèves de décrire la fréquence à laquelle le ou les parents écoutent de la musique classique avec l'enfant, la fréquence à laquelle ils prennent le repas principal tous ensemble, celle à laquelle le ou les parents consacrent du temps à parler avec l'enfant et discutent avec l'enfant de l'actualité politique ou sociale, de livres ou de films ainsi que des résultats scolaires obtenus par l'enfant.

- Pour définir la variable "activités culturelles", les élèves précisent la fréquence à laquelle ils vont au cinéma, vont voir une pièce de théâtre, assistent à un événement sportif, visitent un musée ou une galerie d'art, ou assistent à un concert de musique classique, un opéra ou un ballet.

- Pour évaluer l'apport de l'aide aux devoirs, les élèves fournissent la fréquence à laquelle ils reçoivent de l'aide de leur mère, de leur père, de leurs frères ou sœurs, grands-parents, autres connaissances ou amis de leurs parents pour leur travail scolaire.

- Pour définir une variable "ressources éducatives et culturelles», les élèves indiquent s'ils possèdent un bureau, une chambre à eux seuls, un endroit calme où travailler, un ordinateur, des logiciels éducatifs, une calculatrice à leur seul usage, de la littérature classique chez eux, des œuvres d'art, un dictionnaire, etc.

- Pour préciser "profession future attendue», les élèves sont invités à mentionner le type de métier qu'ils espèrent pratiquer lorsqu'ils auront environ 30 ans.

\section{Survol de la méthode d'estimation par quantiles}

Le but de la présente analyse est d'estimer l'effet de différentes variables, mesurées dans le cadre de la collecte de données internationale PISA 2000, sur la performance scolaire d'élèves au Canada. La contribution principale de notre étude consiste en la comparaison des effets estimés sur la performance des élèves pour différents quantiles de la variable dépendante. C'est-à-dire que nous étudions non seulement une diversité de niveaux socioéconomiques, mais aussi leur relation selon le quantile de la variable dépendante. Les quantiles sont des points de la distribution de probabilité d'une variable aléatoire. Dans la présente étude, la variable en question est la variable dépendante (la réussite en littératie) que nous souhaitons expliquer, et les quantiles sont plus précisément les centiles. Pour illustrer, considérons le $50^{\mathrm{e}}$ centile. Celui-ci correspond simplement à la médiane de la distribution. Un élève dont la performance est au $50^{\mathrm{e}}$ centile réussit mieux que $50 \%$ des élèves dans l'échantillon, et moins bien que $50 \%$ des élèves. Pour cette étude, nous étudions les $5^{\mathrm{e}}$, $25^{\mathrm{e}}, 50^{\mathrm{e}}, 75^{\mathrm{e}}$ et $95^{\mathrm{e}}$ centiles. Cela nous permet d'établir les différences entre les élèves de divers niveaux de réussite, indépendamment de leur milieu socioéconomique. 
Afin de mieux illustrer l'utilité des quantiles, nous proposons d'estimer d'abord un modèle linéaire de régression multivariée, puis nous estimons un modèle semblable dans le cadre d'une régression par quantiles. L'objectif est de tenter d'élucider le faible lien entre les variables indépendantes liées aux ressources de l'école et la variable dépendante, c'est-à-dire une mesure de performance scolaire chez l'élève (Hanushek, 1996). L'hypothèse posée est: si les ressources de l'école ont un effet très différent sur les élèves selon leur niveau de réussite, une analyse qui considère seulement l'élève moyen pourrait être portée à conclure que l'effet est très faible, alors qu'il pourrait être au contraire important pour certains groupes d'élèves, par exemple ceux et celles qui ont plus de difficulté scolaire.

La régression linéaire est une approche utile pour dresser un lien entre variable dépendante et variables indépendantes d'après un modèle qui est justifié par la théorie. Le modèle canonique se présente comme suit:

$$
\mathrm{y}_{\mathrm{i}}=\mathrm{b}_{0}+\mathrm{b}_{1} \mathrm{x}_{1, \mathrm{i}}+\mathrm{b}_{2} \mathrm{x}_{2, \mathrm{i}}+\mathrm{b}_{3} \mathrm{x}_{3, \mathrm{i}}+\mathrm{e}_{\mathrm{i}}
$$

où y est la variable dépendante, par exemple une mesure de réussite scolaire, où chacun des $x$ est une variable indépendante (dans cet exemple il y en a trois), où $e$ est une variable-erreur aléatoire qui prend en moyenne une valeur de zéro et où les indices $i$ représentent une observation particulière (un individu) dans l'échantillon. La théorie suggère des hypothèses portant sur les valeurs que prennent les divers $b_{1}, b_{2}, b_{3}$, par exemple l'hypothèse qu'un certain $b$ soit zéro ou que la somme de deux $b$ soit un.

La régression par quantiles permet d'estimer les valeurs de $b$ conditionnellement à la valeur de la variable dépendante $y$, par exemple si cette dernière est au $75^{\mathrm{e}}$ ou au $95^{\mathrm{e}}$ centile. Dans le cadre de la présente étude, nous évaluons par exemple les différences pour un groupe de variables indépendantes entre les élèves de milieux défavorisés qui réussissent très bien et ceux et celles qui réussissent moins bien.

La littérature empirique sur la question de l'effet sur les élèves des variables liées à l'école est vaste et les conclusions, partagées (Fertig, 2003; Hanushek, 1996; Case et Deaton, 1999. La présence de ces difficultés méthodologiques encourage l'utilisation d'une nouvelle approche. Eide et Showalter (1998) par exemple étudient les facteurs qui influencent la qualité des écoles. Ils observent que la régression par quantiles offre des résultats plus riches et nuancés. Ainsi, notre hypothèse consiste à reconnaître que, pour mieux comprendre les facteurs qui aident les élèves de milieux défavorisés, il est souhaitable d'estimer les effets de façon conditionnelle en tenant compte du niveau de réussite des élèves. 


\section{Résultats pour les élèves issus de milieux défavorisés}

\section{Résultats pour la régression par quantiles appliquée au sous- échantillon d'élèves de milieux défavorisés, $95^{\circ}$ centile de réussite, mesurée par la valeur plausible PISA}

Il est à noter que dans le reste du texte «significatif » sera employé au même sens que «statistiquement significatif». En outre, toutes les variables qui sont discutées dans l'interprétation des résultats sont significatives. De plus, les dimensions interactive (I), normative (N) et structurale (S) ont été précisées pour chacune des variables statistiquement significatives.

Le sous-échantillon analysé ici considère les élèves de milieux défavorisés dont la performance sur le test PISA est supérieure à $95 \%$ des résultats des autres élèves du même milieu. Dans un premier temps, il est possible de reconnaître que le nombre de variables statistiquement significatives est plus grand pour les filles que pour les garçons. Cela peut suggérer qu'il est plus difficile d'identifier des variables qui peuvent affecter la performance des garçons sur le test de lecture de PISA que ce ne l'est pour les filles.

Tableau 1. Analyse par quantiles (régression pour le $95^{\mathrm{e}}$ centile de réussite sur le test PISA tel que mesuré par la valeur plausible), sous-échantillon élèves défavorisés

\begin{tabular}{|c|c|c|c|}
\hline Dimension & Dimen & & Valeur plausible $95^{\mathrm{e}}$ centile \\
\hline & Effet positif ou association positive & & Effet négatif ou association négative \\
\hline I & Joie de lire & $\mathrm{N}$ & Appui éducationnel des parents": \\
\hline I & Habiletés informatiques* & $\mathrm{N}$ & Sens d'appartenance*: \\
\hline$S$ & Avoirs financiers*: & & \\
\hline 1 & Communication culturelle & & \\
\hline $\mathrm{S}$ & $\begin{array}{l}\text { Manque de personnel enseignant } \\
\text { en sciences : }\end{array}$ & & \\
\hline$S$ & Absence d'enseignants est un problème & & \\
\hline & Garçons & & Valeur plausible $95^{\mathrm{e}}$ centile \\
\hline & Effet positif ou association positive & & Effet négatif ou association négative \\
\hline 1 & Profession future attendue* & $\mathrm{N}$ & Appui éducationnel des parents" \\
\hline 1 & Lien enseignant-élève* & $\mathrm{N}$ & $\begin{array}{l}\text { Appui des enseignants et } \\
\text { enseignantes" }\end{array}$ \\
\hline
\end{tabular}

Estimations des paramètres associés avec les variables indépendantes, regroupés par direction (effet positif ou négatif), tous significatifs au seuil de $1 \%\left({ }^{* *}\right)$ ou de $5 \%(")$.

Selon le modèle de création d'un capital humain chez les élèves issus de milieux défavorisés (Zhang et al. 2008), il est possible de regrouper certaines variables. Ainsi, sous l'influence de la famille, «les avoirs financiers» et la «communication culturelle» apparaissent significatifs au même seuil et en même temps que les variables «habiletés informatiques» et "joie de lire», que nous situons sous l'influence de 
l'école. L'étude de Cézard, Gollac et Rougerie (2000) montre l'importance du capital culturel dans l'utilisation de l'informatique dans le milieu du travail puisque l'ordinateur est un outil de travail et un bien culturel. Ainsi, la variable «avoirs financiers » de la famille pourrait expliquer le développement des "habiletés informatiques» pour les élèves de ce quantile. En outre, la communication culturelle alimenterait la variable «joie de lire». Ainsi, considérées du point de vue de l'accès à l'écrit, ces variables sont liées entre elles, ce qui ajoute à leur importance. Par ailleurs, les variables «manque d'enseignants en science» et «absences des enseignants» sont corrélées positivement pour les élèves issus de milieux défavorisés qui réussissent au $95^{\mathrm{e}}$ centile, des variables que nous regroupons sous l'influence de l'école. Dans ces conditions, nous posons l'hypothèse qu'en l'absence d'enseignants les élèves sont poussés à avoir plus d'autonomie dans la réalisation de leurs travaux et, par conséquent, à lire davantage. Cette hypothèse se voit renforcée par la présence de la variable «joie de lire» que nous avons déjà discutée. La variable «sens d'appartenance» est corrélée négativement, ce qui semble contredire les résultats obtenus lors d'une analyse réalisée précédemment (DeBlois, Rousseau et Lapointe, 2007). Il faut toutefois retenir que ces résultats sont vrais seulement pour les filles, qui réussissent bien au-dessus de la moyenne, donc celles qui n'ont pas besoin de ce soutien, ce qui confirmerait l'importance de l'autonomie de ces élèves.

Il semble que peu de variables puissent être identifiées à travers les questionnaires de PISA 2000 pour les garçons issus de ces milieux par rapport aux élèves favorisés (voir l'annexe). Pour ceux qui réussissent au $95^{\mathrm{e}}$ centile, seules les variables "profession future attendue» et «lien enseignant-élève» sont corrélées positivement. Ces variables sont différentes de celles des filles. En outre, une corrélation négative apparaît pour les variables « appui éducationnel familial» et «appui de l'enseignant». Nous expliquons ce résultat par le fait que les élèves ayant besoin d'aide vont solliciter les parents ou les enseignants, ce qui n'est pas le cas de ce groupe.

En somme, pour les filles et les garçons issus de milieux défavorisés qui réussissent très bien, il semble que les variables liées à une dimension interactive soient corrélées positivement, alors que celles qui sont liées à une dimension normative sont corrélées négativement.

\section{Résultats pour la régression par quantiles appliquée au sous- échantillon $d^{\prime}$ élèves de milieux défavorisés, $75^{\mathrm{e}}$ centile de réussite, mesurée par la valeur plausible PISA}

Pour les filles dont le niveau de réussite en lecture, mesuré par PISA, est plus fort que $75 \%$ des résultats des autres élèves du même milieu socioéconomique, la variable du niveau d'éducation des parents, que nous avons l'habitude de rencontrer dans ce type d'étude, montre de nouveau des corrélations positives. Cette variable s'accompagne de celles qui ont été observées précédemment: «habiletés informatiques» et «joie de lire». Les variables «communication culturelle» et «avoirs financiers» de la famille sont encore présentes. Enfin, la "profession future attendue» qui était présente pour les garçons apparaît pour les filles. Elle sera présente à chacun des centiles inférieurs à 75 par la suite. 
Tableau 2. Analyse par quantiles (régression pour le $75^{\mathrm{e}}$ centile de réussite sur le test PISA tel que mesuré par la valeur plausible), sous-échantillon élèves défavorisés

\begin{tabular}{|c|c|c|c|}
\hline \multirow[t]{2}{*}{ Dimension } & Filles & ion & Valeur plausible $75^{\mathrm{e}}$ centile \\
\hline & \multicolumn{2}{|l|}{ Effet positif ou association positive } & Effet négatif ou association négative \\
\hline 1 & Profession future attendue & $\mathrm{N}$ & Appui éducationnel des parents \\
\hline$S$ & $\begin{array}{l}\text { Maximum du niveau d'éducation des } \\
\text { parents:" }\end{array}$ & $\mathrm{N}$ & Sens d'appartenance \\
\hline 1 & Joie de lire & $\mathrm{N}$ & Pression pour réussir:* \\
\hline 1 & Habiletés informatiques: & $S$ & Possessions culturelles: \\
\hline$S$ & Avoirs financiers:: & $\mathrm{N}$ & Attitude à l'égard de l'informatique: \\
\hline \multirow[t]{4}{*}{ I } & \multirow[t]{2}{*}{ Communication culturelle*: } & S & Bâtiments en pauvre condition" \\
\hline & & $\mathrm{N}$ & École buissonnière est un problème \\
\hline & Garçons & & Valeur plausible $75^{\mathrm{e}}$ centile \\
\hline & Effet positif ou association positive & & Effet négatif ou association négative \\
\hline 1 & Profession future attendue & $\mathrm{N}$ & Appui éducationnel des parents \\
\hline I & Habiletés informatiques* & $\mathrm{N}$ & Expérience informatique* \\
\hline 1 & Lien enseignant-élève* & & \\
\hline
\end{tabular}

Les variables ayant une corrélation négative sont les mêmes que pour le $95^{\mathrm{e}}$ centile, mais d'autres s'ajoutent. Ainsi, la variable "pression pour réussir» nous apprend que moins les filles ont de pression, meilleurs sont leurs résultats. Toutefois, cette variable est présente aussi chez les filles qui réussissent moins bien. Il n'est donc pas possible de tirer de conclusion nette. Toutefois, cette variable n'est pas présente lorsque les filles réussissent au $95^{\mathrm{e}}$ centile, ce qui semble s'expliquer par la confiance que ces dernières ont acquise lorsqu'elles réussissent. En outre, la variable «possessions culturelles" offre aussi une corrélation négative. Cela nous conduit à interpréter que ce ne sont pas les possessions culturelles qui influencent les résultats des filles en lecture mais bien ce qui se fait avec ces livres ou ces films.

Pour les garçons, les variables «habiletés informatiques» et «lien enseignantélève", que nous regroupons sous l'influence de l'école, offrent des corrélations positives. Les habiletés informatiques développées confirment ainsi l'accès à l'écrit par ce véhicule. Sous l'influence de la famille, la «profession future attendue» offre une corrélation positive. Toutefois, l'«appui éducationnel familial» et l'«expérience informatique» présentent de nouveau une corrélation négative.

En somme, les variables liées à la dimension interactive montrent des corrélations positives tant chez les garçons que chez les filles. Alors que les filles qui se situent dans le $75^{\mathrm{e}}$ et le $95^{\mathrm{e}}$ centile semblent plus influencées par la variable «communications culturelles», chez les garçons ce sont les variables «lien enseignantélève» et «profession future attendue» qui sont associées à la réussite en lecture. 
Dans ces conditions, le rôle des modèles de trajectoire de vie (les mentors) aurait une importance particulière pour les garçons. Toutefois, les variables liées à une dimension normative présentent à nouveau une corrélation négative.

\section{Résultats pour la régression par quantiles appliquée au sous- échantillon d'élèves de milieux défavorisés, $50^{\circ}$ centile de réussite (médiane), mesurée par la valeur plausible PISA}

Il est intéressant de noter que le nombre de variables significatives est plus grand pour les centiles vers le centre de la distribution que pour les centiles aux deux extrêmes ( $95^{\mathrm{e}}$ et $5^{\mathrm{e}}$ centiles). Deux explications sont possibles. Il se peut que les élèves aux deux extrêmes de la distribution soient peu sensibles aux interventions ou influences diverses, positives ou négatives. Ainsi, une stratégie pourrait être bénéfique pour l'élève moyen sans nécessairement avoir d'impact sur les élèves particulièrement forts ou faibles. Toutefois, une seconde possibilité est que d'un point de vue statistique, comme il y a moins d'élèves dans les extrêmes que dans le centre de la distribution, il est plus difficile pour les premiers d'obtenir des coefficients significatifs que pour les derniers. Dans ce cas, nous avons pour résultat un nombre plus petit de variables significatives.

Tableau 3. Analyse par quantiles (régression pour le $\mathbf{5 0}^{\mathrm{e}}$ centile de réussite (la médiane) sur le test PISA tel que mesuré par la valeur plausible), souséchantillon élèves défavorisés

\begin{tabular}{|c|c|c|c|}
\hline Dimension & Filles & Dimension & Valeur plausible $50^{\mathrm{e}}$ centile \\
\hline & Effet positif ou association positive & & Effet négatif ou association négative \\
\hline 1 & Profession future attendue & $\mathrm{N}$ & Appui éducationnel des parents \\
\hline$S$ & $\begin{array}{l}\text { Maximum du niveau d'éducation } \\
\text { des parents }\end{array}$ & $\mathrm{N}$ & Sens d'appartenance: \\
\hline I & Joie de lire & $\mathrm{N}$ & Pression pour réussir: \\
\hline 1 & Habiletés informatiques" & $\mathrm{N}$ & Expérience informatique* \\
\hline$S$ & Avoirs financiers & $S$ & Bâtiments en pauvre condition* \\
\hline $\mathrm{N}$ & $\begin{array}{l}\text { Autonomie de l'enseignant ou } \\
\text { de l'enseignante: }\end{array}$ & & \\
\hline$S$ & Infrastructure physique de l'école & & \\
\hline & Garçons & & Valeur plausible $75^{\mathrm{e}}$ centile \\
\hline & Effet positif ou association positive & & Effet négatif ou association négative \\
\hline 1 & Profession future attendue & $\mathrm{N}$ & Soutien scolaire des parents \\
\hline 1 & Habiletés informatiques" & $S$ & Possessions culturelles \\
\hline & & $\mathrm{N}$ & Expérience informatique: \\
\hline & & $\mathrm{N}$ & Appui des enseignants: \\
\hline
\end{tabular}

Estimations des paramètres associés avec les variables indépendantes, regroupés par direction (effet positif ou négatif), tous significatifs au seuil de $1 \%(* *)$ ou de $5 \%(")$. 
Pour les filles dont le niveau de réussite mesuré par PISA est plus fort que $50 \%$ des résultats des autres élèves du même milieu, le niveau maximum d'éducation des parents et les avoirs financiers de la famille sont des variables qui offrent une corrélation positive. Les variables «autonomie de l'enseignant» et «infrastructure physique de l'école», qui sont sous l'influence de l'école, s'ajoutent. Les variables «joie de lire» et «habiletés informatiques» offrent à nouveau des corrélations positives. Certaines variables comme «expérience informatique» et «condition physique médiocre des bâtiments» sont corrélées négativement. Sous l'influence de la famille, les variables dont la corrélation est négative sont «appui éducationnel familial» et «pression pour réussir ». Ces résultats sont assez semblables à ceux observés au $75^{\mathrm{e}}$ centile, mais les élèves réussissent moins bien. Ainsi, plusieurs variables semblent intervenir pour une plus grande quantité d'élèves.

Pour les garçons, les variables, sous l'influence de la famille, dont la corrélation est positive sont les «habiletés informatiques» et la "profession future attendue». Certaines variables, sous l'influence de l'école, offrent toujours une corrélation négative. C'est le cas de l'«expérience informatique» et de l'«appui des enseignants». Ce même type de variables, sous l'influence de la famille, présentent aussi une corrélation négative. Il s'agit de l'«appui éducationnel familial» et des "possessions culturelles ». La récurrence de ces corrélations tend à rendre de plus en plus plausibles les interprétations réalisées précédemment.

En somme, les variables de la dimension interactive présentent généralement une corrélation positive tant pour les garçons que pour les filles. Toutefois, les variables de la dimension structurale semblent plus importantes pour les filles que pour les garçons. Enfin, les variables de la dimension normative offrent de nouveau des corrélations négatives.

\section{Résultats pour la régression par quantiles appliquée au sous- échantillon d'élèves de milieux défavorisés, $25^{\mathrm{e}}$ centile de réussite, mesurée par la valeur plausible PISA}

Rappelons que le sous-échantillon analysé considère les élèves de milieux défavorisés dont la performance sur le test PISA est supérieure à $25 \%$ des résultats des autres élèves issus du même milieu. 
Tableau 4. Analyse par quantiles (régression pour le $25^{\mathrm{e}}$ centile de réussite sur le test PISA tel que mesuré par la valeur plausible), sous-échantillon élèves défavorisés

\begin{tabular}{|c|c|c|c|}
\hline \multirow[t]{2}{*}{ Dimension } & Filles & Dimension & Valeur plausible $25^{\mathrm{e}}$ centile \\
\hline & \multicolumn{2}{|l|}{ Effet positif ou association positive } & Effet négatif ou association négative \\
\hline 1 & Profession future attendue: & $\mathrm{N}$ & Appui éducationnel des parents \\
\hline 1 & Joie de lire & $\mathrm{N}$ & Pression pour réussir:*: \\
\hline I & Habiletés informatiques: & $\mathrm{N}$ & Expérience informatique \\
\hline$S$ & $\begin{array}{l}\text { Maximum du niveau d'éducation } \\
\text { des parents }\end{array}$ & $S$ & Bâtiments en pauvre condition* \\
\hline$S$ & Avoirs financiers" & S & $\begin{array}{l}\text { Insuffisance du temps } \\
\text { d'enseignement }\end{array}$ \\
\hline \multirow[t]{3}{*}{$S$} & Ressources éducatives de la famille* & $S$ & $\begin{array}{l}\text { Qualité des ressources éducatives de } \\
\text { l'école, d'après l'administration:" }\end{array}$ \\
\hline & \multicolumn{2}{|l|}{ Garçons } & Valeur plausible $75^{\mathrm{e}}$ centile \\
\hline & \multicolumn{2}{|l|}{ Effet positif ou association positive } & Effet négatif ou association négative \\
\hline 1 & Profession future attendue & $\mathrm{N}$ & Appui éducationnel des parents \\
\hline $\mathrm{S}$ & $\begin{array}{l}\text { Maximum du niveau d'éducation } \\
\text { des parents }\end{array}$ & $S$ & Possessions culturelles* \\
\hline$S$ & Ressources éducatives du ménage: & $\mathrm{N}$ & Expérience informatique \\
\hline$S$ & Matériel de laboratoire insuffisant* & $S$ & $\begin{array}{l}\text { École financée en partie par frais de } \\
\text { scolarité* }\end{array}$ \\
\hline $\begin{array}{l}\text { Estim } \\
\text { (effet }\end{array}$ & $\begin{array}{l}\text { S paramètres associés avec les vari } \\
\text { u négatif), tous significatifs au seui }\end{array}$ & $\begin{array}{l}\text { riables in } \\
\text { uil de } 1 \%\end{array}$ & $\begin{array}{l}\text { pendantes, regroupés par direction } \\
\text { ) ou de } 5 \%\left({ }^{(*)} \text {. }\right.\end{array}$ \\
\hline
\end{tabular}

À nouveau, la variable «maximum du niveau d'éducation des parents» s'ajoute la variable «ressources éducatives de la famille», et ce, tant pour les garçons que pour les filles. La variable "ressources éducatives de la famille» n'est présente qu'au $25^{\mathrm{e}}$ centile, aussi bien chez les garçons que les filles. La variable "avoirs financiers" demeure présente au $25^{\mathrm{e}}$ centile pour les filles. Les variables «habiletés informatiques», «joie de lire» et «profession future attendue» offrent des corrélations positives.

Chez les garçons, seule la variable "profession future attendue» montre une corrélation positive. La corrélation positive de la réussite en lecture avec la variable «matériel de laboratoire insuffisant» pourrait être interprétée comme une occasion ratée de contextualiser les activités de lecture, d'où les moins bons résultats des élèves.

Déjà, nous observions la présence de corrélations significatives et positives pour des variables liées à la famille. Toutefois, ces dernières ne permettent pas aux élèves d'obtenir de meilleurs résultats au test de lecture de l'enquête PISA 2000. La dimension structurale s'ajoute de façon importante. Les variables liées à la dimension normative ne présentent que des corrélations négatives. 
Résultats pour la régression par quantiles appliquée au souséchantillon $\mathbf{d}^{\prime}$ élèves défavorisés, $5^{\mathrm{e}}$ centile de réussite, mesurée par la valeur plausible PISA

Rappelons que la performance d'un élève au $5^{\mathrm{e}}$ centile de réussite selon le test PISA 2000 serait plus faible que $95 \%$ de tous les résultats parmi les élèves dans cet échantillon.

Tableau 5 . Analyse par quantiles (régression pour le $\mathbf{5}^{\mathbf{e}}$ centile de réussite sur le test PISA tel que mesuré par la valeur plausible), sous-échantillon élèves défavorisés filles et garçons pris séparément

\begin{tabular}{|c|c|c|c|}
\hline Dimension & Filles & & Valeur plausible $5^{\mathrm{e}}$ centile \\
\hline & \multicolumn{2}{|l|}{ Effet positif ou association positive } & Effet négatif ou association négative \\
\hline I & Profession future attendue de l'élève & $S$ & $\begin{array}{l}\text { Qualité des ressources éducatives de } \\
\text { I'école, d'après l'administration" }\end{array}$ \\
\hline \multirow[t]{3}{*}{1} & Joie de lire* & $S$ & $\begin{array}{l}\text { Disponibilité des programmes de } \\
\text { formation du personnel enseignant: }\end{array}$ \\
\hline & Garçons & & Valeur plausible $75^{\mathrm{e}}$ centile \\
\hline & Effet positif ou association positive & & Effet négatif ou association négative \\
\hline I & Profession future attendue de l'élève ${ }^{* * *}$ & $S$ & Absence de ressources multimédias: \\
\hline 1 & Habiletés informatiques" & & \\
\hline
\end{tabular}

Dans ce sous-échantillon d'élèves, les seules variables dont les coefficients sont positifs pour les filles sont «profession future attendue de l'élève» et «joie de lire», des variables liées à la dimension interactive. Les variables "programmes de développement carrière enseignants" et "ressources éducatives de l'école» offrent des corrélations négatives. Comme il s'agit d'une association (corrélation) et non pas nécessairement d'une relation cause à effet, l'interprétation raisonnable de ce résultat est que, lorsque les élèves ont beaucoup de difficulté, les écoles vont chercher davantage de ressources éducatives et les enseignants et enseignantes vont chercher davantage de formation.

Chez les garçons, les variables «profession future attendue de l'élève» et «habi letés informatiques», des manifestations de la dimension interactive offrent des corrélations positives. Toutefois, la variable «absence de ressources multimédias » a une corrélation négative. Ainsi, les ressources informatiques et les média seraient bénéfiques pour les élèves en grande difficulté dans la mesure où ils les introduisent à l'écrit et à une variété d'informations. 


\section{Relation entre les variables et les composantes du modèle de création du capital humain de l'élève (Zhang et al., 2008)}

Les résultats sont en général plus riches pour les filles que pour les garçons et semblent moins informatifs aux extrêmes, c'est-à-dire au $5^{\mathrm{e}}$ et au $95^{\mathrm{e}}$ centile. Par exemple, les élèves pour lesquels les résultats obtenus au test PISA 2000 sont plus faibles que $95 \%$ de tous les résultats parmi les élèves de milieux défavorisés montrent peu de corrélations avec les variables évaluées par le questionnaire de l'élève PISA 2000. Des cadres théoriques sociologiques, psychopédagogiques, psychologiques ou didactiques permettraient d'introduire de nouvelles variables.

Le modèle de création du capital humain (Zhang et al., 2008) considère les environnements familial, scolaire et communautaire. La recherche en éducation portant sur le capital social se concentre sur le développement de capital social et financier comme déterminants du capital humain. Relativement peu d'études portent autant d'attention sur les différentes dimensions du capital social et leurs rôles potentiellement différents pour la création de capital humain chez l'enfant. Le regroupement de l'ensemble des variables à l'étude en trois dimensions (structurale, normative et interactive) a conduit à certaines observations.

Ainsi, lorsque le niveau de réussite mesuré par PISA 2000 est plus élevé que $75 \%$ des résultats des autres élèves issus de milieux défavorisés, les variables de la dimension interactive semblent importantes tant pour les garçons que pour les filles. De plus, les variables qui apparaissent pour les filles qui se situent au-dessus du $50^{\mathrm{e}}$ percentile de réussite (la médiane) introduisent la dimension structurale tant du point de vue de l'école que de la famille. Par exemple, les variables «niveau maximum d'éducation des parents" et "avoirs financiers de la famille» offrent des corrélations positives tout comme les variables «autonomie des enseignants» et «infrastructure scolaire».

Enfin, pour les filles dont le niveau de réussite est plus faible que $75 \%$ des autres élèves, les dimensions structurales et interactives dominent alors que la dimension normative offre toujours des effets ou associations négatifs. Ces dernières variables ont en commun d'offrir soit une forme d'appui ou une forme de pression. Une interprétation raisonnable de ces liens négatifs serait que les filles qui ont plus de difficulté vont recevoir davantage d'appui, ce qui crée davantage de pression. Toutefois, cela n'améliore pas les résultats au test de lecture. Une deuxième hypothèse voudrait que les interventions portent davantage sur les comportements des élèves au détriment d'interventions sur les contenus d'apprentissage. Une troisième hypothèse consiste à penser que les interventions d'apprentissage portent davantage sur des stratégies d'enseignement qui font intervenir la répétition d'explications plutôt que l'enrichissement des conceptualisations. René de Cotret et Giroux (2003) ont d'ailleurs observé comment le même enseignant modifiait son intervention devant des classes de doubleurs.

La plupart des études quantitatives empiriques ne peuvent déceler de lien statistiquement significatif entre les variables liées aux écoles et aux diverses mesures de réussite académique (Hanushek, 1986, 1996). Le modèle de la création du capital humain de l'élève permet de structurer les variables afin d'étudier les relations entre 
elles. Ces observations conduisent à discuter le modèle initial pour y situer l'influence de l'élève dans la constitution des différentes dimensions. Dans ces conditions, ce modèle permettrait de soulever de nouvelles questions compte tenu de la présence simultanée de plusieurs variables et de la posture proactive de l'élève.

\section{Résultats et discussion}

Notre objectif dans cette étude était de contribuer à identifier des éléments de réponse aux questions énoncées au départ en confirmant ou en infirmant l'hypothèse selon laquelle le capital humain et financier de l'école pouvait compenser le niveau socioéconomique moins élevé de la famille pour observer une relation statistiquement significative et positive pour au moins certaines variables. L'analyse par quantile permet d'observer une corrélation entre certaines variables telles que la «joie de lire» ou encore la "profession attendue de l'élève». La réussite des élèves, pour tous les quantiles, montre aussi l'importance de l'engagement de l'élève dans le développement de son capital humain. Ce résultat laisse voir l'importance d'introduire l'influence proactive de l'élève dans le modèle.

Schéma 2. Modèle modifié de création, à travers le capital social, d'un capital humain chez les élèves issus de milieux défavorisés

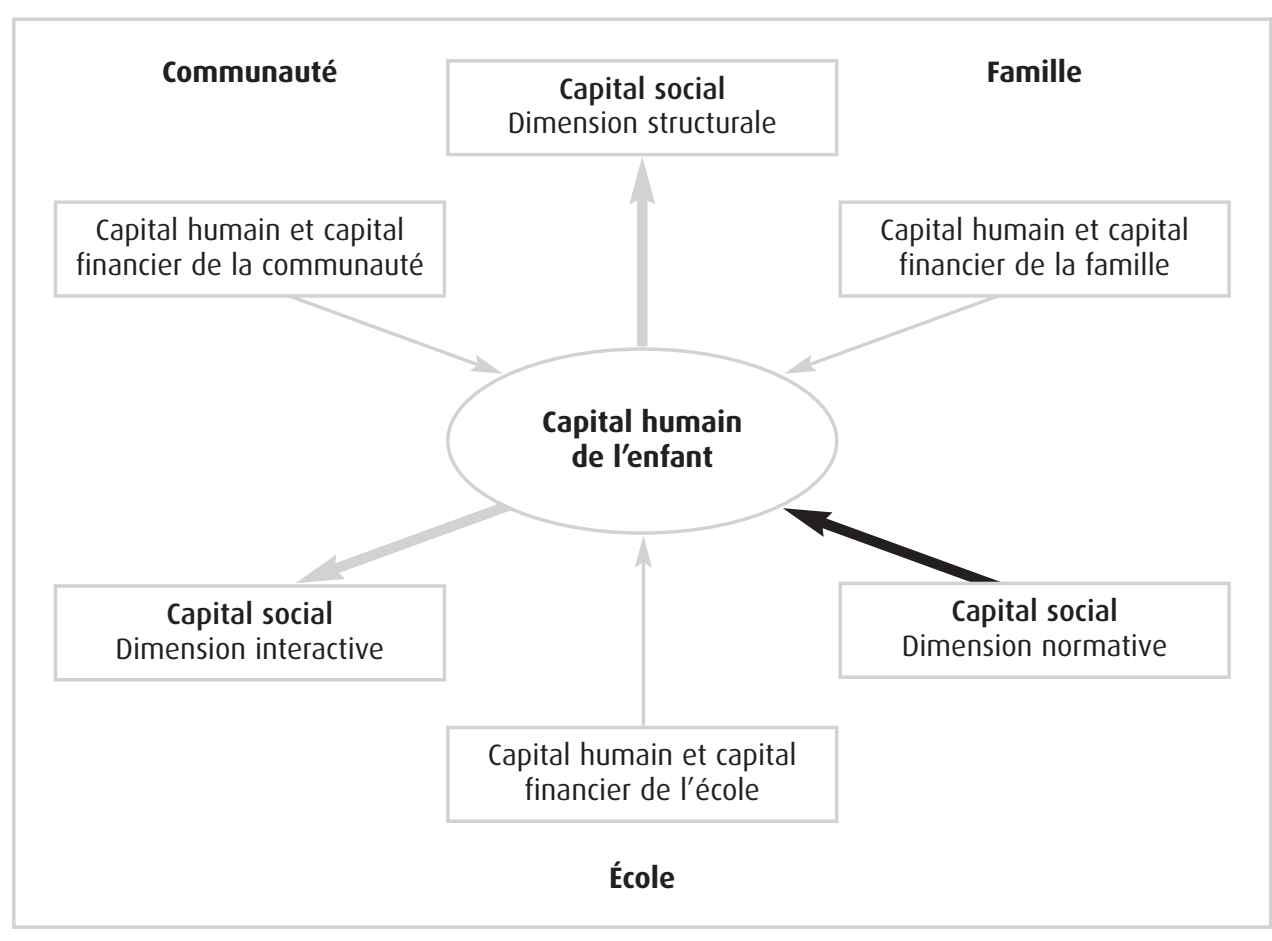


Le modèle initial a permis d'organiser les données, plus particulièrement en ce qui concerne les facteurs familiaux et les facteurs scolaires. Toutefois, en inversant la direction des flèches centrales et en établissant une relation entre le capital humain de l'élève et les dimensions structurale et interactive, nous offrons à l'élève une posture proactive dans la définition des différentes dimensions du capital social. La dimension normative demeure cependant orientée dans la même direction en raison de la corrélation négative observée entre les variables en jeu et la réussite en lecture. Étant donné que cette dimension correspond au sentiment d'appartenance, à la pression de réussite, aux appuis des parents et des enseignants, la constitution de ce type réseau social ne serait pas adaptée pour favoriser la réussite en lecture. Ce choix oriente notre interprétation des résultats.

L'analyse selon la régression par quantiles montre que l'effet de diverses variables liées à l'école n'est pas le même pour les élèves de milieux défavorisés qui réussissent bien et les élèves de même milieu qui ont de la difficulté. De plus, il semble que les variables liées à l'école sont plus souvent significatives pour les filles que pour les garçons. En outre, les variables liées à l'école semblent habituellement imbriquées dans la dimension interactive. L'exemple de la variable «lien enseignantélève» permet de réfléchir à cette question. Ainsi, la relation entre enseignant et élève semble particulièrement importante pour les garçons des $75^{\mathrm{e}}$ au $95^{\mathrm{e}}$ centile. Le rôle de l'enseignant est important tant pour les garçons que pour les filles, mais de façon différente. Par exemple, la réussite chez les garçons semble influencée en particulier par les interactions. Par contre, la réussite des filles serait affectée davantage par des variables de la dimension structurale, telles que "autonomie de l'enseignant", «manque problématique d'enseignants», «absence problématique d'enseignants", «insuffisance problématique de temps d'enseignement», qui ne sont pas significatives pour les garçons. (À noter aussi que pour les filles la variable «moral de l'enseignant» est présente, mais elle n'est significative qu'au seuil de $10 \%$. Elle n'est donc pas présentée dans les résultats.) Ces variables pourraient engendrer la nécessité, pour les filles, de développer leur autonomie, ce qui est socialement valorisé.

En ce qui concerne la famille, les «avoirs financiers» ont une corrélation positive beaucoup plus importante pour les filles que pour les garçons, et ce lien est plus fort parmi les élèves résilients que parmi les élèves en grande difficulté. En outre, la variable «maximum de niveau d'éducation» des parents est corrélée positivement pour les filles et pour les garçons, sauf aux extrêmes. Toutefois, «l'appui éducationnel de la famille» offre une corrélation négative à tous les quantiles, ce qui vient confirmer nos travaux portant sur l'aide aux devoirs (DeBlois et al., 2009).

Toutefois, bien qu'importantes, l'ensemble des variables à l'étude ne permettent pas de discriminer des interventions spécifiques pour cette population. En effet, nous observons l'importance de la dimension interactive par la présence de la variable «profession future attendue de l'élève » à tous les quantiles pour les garçons et les filles, sauf au $95^{\mathrm{e}}$ pour les filles. Cette variable n'est donc pas discriminante au sens où elle est présente chez l'ensemble des élèves interrogés, mais elle demeure importante pour tous les élèves. La variable «joie de lire», pour sa part, est corrélée positivement avec la réussite en lecture à tous les quantiles pour les filles, mais 
n'apparaît pas pour les garçons puisqu'elle se manifeste par la variable «habiletés informatiques». Cette variable devient donc discriminante pour les filles seulement. Nous avons déjà observé des différences plus grandes entre les filles et les garçons, et ce, entre les élèves au Québec et pour l'ensemble des élèves canadiens (Rousseau, DeBlois, Lapointe, 2008). Nos résultats invitent à la prudence, puisque ces profils d'apprenants différenciés selon le sexe pourraient varier selon les contextes sociaux et culturels. Pour être en mesure de généraliser ces constats, il demeure important d'étudier la question par contexte socioculturel.

Enfin, le nombre de variables sur lesquelles il semble possible d'intervenir est moins élevé chez les élèves issus de milieux défavorisés que chez l'ensemble des élèves. Il semble donc y avoir très peu de variables au $5^{\mathrm{e}}$ centile, parmi celles identifiées par les questionnaires Pisa 2000, sur lesquelles il est possible d'observer des corrélations avec la réussite à ce test lorsque les élèves sont issus de milieux défavorisés. Pour l'ensemble des élèves, tant pour les garçons que pour les filles, les variables communes sont liées à la dimension interactive (la communication culturelle, le plaisir de lire, les relations enseignant-élèves et les habiletés informatiques, la profession attendue de l'élève). Ces variables, considérées comme des charnières, pourraient servir d'interfaces entre l'école et la famille. Elles pourraient contribuer à la création d'interactions entre l'élève, l'enseignant et les parents pour favoriser le développement d'un capital scolaire évoqué dans les études de Moatty (1995). En effet, pour lui, la formation, un indicateur du capital scolaire joue un rôle important au moment de l'insertion professionnelle, notamment dans le secteur privé où les structures plus petites exigent des qualités sociales et scolaires (Moatty, 2004, p. 94). En outre, de nouveaux comportements culturels, comme la communication et la coopération entre pairs, sont forgés dans la famille ou avec les pairs à l'école. À ce sujet, Dufour et Frimousse (2006) précisent que «le jeune à faible capital scolaire a besoin d'un parrain afin de préparer sa socialisation organisationnelle».

Dans ces conditions, discuter d'une définition de la réussite scolaire devrait non seulement ajouter certaines variables à la dimension normative habituelle mais intégrer une dimension interactive. Ainsi, la réussite scolaire pourrait correspondre non seulement à la réussite des élèves, mais aussi à leur projection dans différents projets, dont celui de leur insertion professionnelle. En outre, la réussite scolaire qui se manifeste à travers des variables liées à la dimension interactive exprime l'engagement de l'élève.

\section{Conclusion}

Un nombre assez grand de variables issues de la base de données PISA 2000 semblent jouer sur la réussite en lecture, sauf au $5^{\mathrm{e}}$ centile. La combinaison des variables liées à la dimension interactive indique l'importance d'offrir aux enseignants et aux parents des conditions qui leur permettent d'utiliser des moyens de collaborer. Enfin, la récurrence de la dimension interactive offre un «lieu» pour créer des interactions entre l'école et la famille et pour vivre la réussite scolaire. 
La méthode d'analyse par quantiles permet de reconnaître l'importance des communications culturelles inscrites dans des relations enseignant-élève, manifestation de la dimension interactive, de même que la variable "profession future attendue par l'élève», manifestation de la dimension interactive, pour favoriser la réussite des élèves issus d'un milieu socioéconomique défavorisé. Ce type de variables pourrait accentuer l'influence de la dimension interactive du modèle initial de Zhang et $a l$. (2008). L'influence de ce modèle sur la définition de la réussite scolaire pourrait offrir une direction pour l'évaluation et l'intervention aux différents niveaux de discours à l'égard de la réussite scolaire.

\section{Références bibliographiques}

BERNARD, B. (1991). Fostering Resiliency in Kids: Protective Factors in the Family, School, and Community. San Francisco: Far West Laboratory for Educational Research and Development.

BERTRAND, R. (2002). L'accès aux ressources pédagogiques: d'autres données en réponse à Zhang. Dans P. de Broucker et A. Sweetman (dir.), Towards Evidencebased Policy for Canadian Education/Vers des politiques canadiennes d'éducation fondées sur la recherche. Montréal et Kingston: McGill-Queen's University/Press for the John Deutsch Institute.

CASE, A. et A. DEATON (1999). School inputs and educational outcomes in South Africa. Quarterly Journal of Economics, 114(3), 1047-1084.

CÉZARD, M., GOLLAC, M. et ROUGERIE, C. (2000). «L'ordinateur, outil de travail et bien culturel», Actes de la recherche en sciences sociales, 134, 22-28.

COLEMAN, J. S. (1988). Social capital in the creation of human capital. The American Journal of Sociology, 94. Supplement: Organizations and Institutions: Social and Economic to the Analysis of Social Structure, S95-S120.

ROUSSEAU, M., DEBLOIS, L. et LAPOINTE, C. (2008). Relations entre le capital familial et le rendement en lecture selon le sexe des élèves. Dans R. Deslandes, Collaboration de l'école, de la famille et de la communauté à l'apprentissage (p. 45-65). Cahiers scientifiques, ACFAS.

DEBLOIS, L., DESLANDES, R., ROUSSEAU, M. et NADEAU, J. (2009). L'accompagnement parental comme facteur de protection de la réussite. Revue McGill. Montréal.

DEBLOIS, L., LAPOINTE, C. et ROUSSEAU, M. (2007, mars). L'équité en éducation. Colloque. Université Laval. www.fse.ulaval.ca/]. 
DUFOUR, L. et FRIMOUSSE, S. (2006). La socialisation organisationnelle des jeunes à faible capital scolaire. Management et avenir, 4(10), 145-160.

EIDE, E. et SHOWALTER, M. H. (XXX). The effect of school quality on student performance: A quantile regression approach. Economic Letters, 58(3), 345-350.

FERTIG, M. (2003). Who's to blame? The determinants of German students' achievement in the PISA 2000 study. IZA Discussion Papers 739. Institute for the Study of Labor (IZA).

GIROUX, J. et RENÉ DE COTRET, S. (2003). Le temps didactique dans trois classes de secondaire I (doubleurs, ordinaires, forts). La spécificité de l'enseignement des mathématiques en adaptation scolaire. Éducation et francophonie, XXXI(2). [En ligne].[http://www.acelf.ca/c/revue/pdf/XXXI_2_155.pdf].

HANUSHEK, E. A. (1996). Measuring investment in education. Journal of Economic Perspectives, 10(4), 9-30.

JACOB, B. et L. LEFGREN (2007). What do parents value in education? An empirical investigation of parents' revealed preferences for teachers. Quarterly Journal of Economics, 122(4), 1603-1637.

KAMANZY, C., ZHANG, X. Y., DEBLOIS, L. et Deniger, M. A. (2007). L'influence du capital social sur la formation du capital humain chez les élèves résilients de milieux socioéconomiques défavorisés. Revue des sciences de l'éducation, XXXIII(1), 127-147.

KNUDSEN, E. I., HECKMAN, J. J., CAMERON, J. L. et SHONKOFF, J. P. (2006). Economic, neurobiological, and behavioral perspectives on building America's future workforce. Proceedings of the National Academy of Sciences, 103, 1015510162.

KOENKER, R. et BASSETT, G. Jr. (1978). Regression quantiles. Econometrica, 46(1), 33-50.

LE MONDE (2007). L'incroyable destinée de Mario Capecchi, Prix Nobel de médecine. Le Monde, 9 octobre, [En ligne]. [http ://www.lemonde.fr/web/article/0,1-0@2-3244,36-965008@51-964090,0.html] (Consulté le 11 novembre 2007).

LEE, C. (2007). From child on the street to Nobel laureate. The Washington Post, 9 octobre, A01.

MASTEN, A. et COATSWORTH, J. D. (1998). The development of competence in favourable and unfavourable environments lessons from research on successful children. American Psychologist, 53(2), 205-220.

MASTEN, A. S. (1994). Resilience in individual development: Successful adaptation despite risk and adversity. Dans M. C. Wang et E. W. Gordon (dir.), Educational Resilience in Inner-City America: Challenges and Prospects (p. 3-25). Hillsdale, NJ : Lawrence Erlbaum. 
MOATTY, Fr. (2004). L'évolution du rôle du capital scolaire dans le modèle sociétal français d'accès à l'informatique au travail: «massification» ou «démocratisation» de l'accès?, Réseaux, 127, 83-114.

NETTLES, S. M. et PLECK, J. H. (1993). Risk, Resilience, and Development: The Multiple Ecologies of Black Adolescents. Baltimore, MD: Center for Research on Effective Schooling for Disadvantaged Students, The Johns Hopkins University.

OCDE (2005). Pisa 2003. Manuel d'analyse des données. Paris: Organisation de coopération et de développement économiques.

RAUDENBUSH, S. W. et WILLMS, J. D. (1991). Schools, Classrooms, and Pupils: International Studies of Schooling from the Multilevel Perspective. New York: Academic Press.

ROTHMAN, R. (1995). Measuring up: Standards, Assessment, and School Reform. San Francisco: Jossey-Bass.

ROUSSEAU, M. et DEBLOIS, L. (2007). L'impact du capital scolaire sur la réussite des élèves en lecture et en mathématiques. Communication présentée dans le cadre des Dixièmes rencontres du Réseau international de recherche en éducation et en formation (REF), Sherbrooke, Québec.

RUTTER, M. (1987). Psychosocial resilience and protective mechanisms. American Journal of Orthopsychiatry, 57(3), 316-331.

STATISTIQUE CANADA (2011). Questions d'éducation: le point sur l'éducation, l'apprentissage et la formation au Canada (Glossaire). Ottawa: Statistique Canada. [En ligne].[http://www.statcan.gc.ca/pub/81-004-x/def/4068716fra.htm] (Consulté le 25 février 2011).

WILLMS, J. D. (2002). The prevalence of vulnerable children. Dans J. D. Willms (dir.), Vulnerable Children: Findings from Canada's National Longitudinal Survey of Children and Youth (p. 45-69). Edmonton: The University of Alberta Press.

ZHANG, X. Y., DEBLOIS, L., KAMANZI, C. et DENIGER, M A. (2008). A theory of success for disadvantaged children: Re-conceptualisation of social capital in the light of resilience. Alberta Journal of Educational Research, 54(1), 97-112.

ZHANG, Y. (2002). The distribution of access to educational resources for 8th grade math in Canada: How equitable is it? Dans P. de Broucker et A. Sweetman (dir.), Towards Evidence-based Policy for Canadian Education/Vers des politiques canadiennes d'éducation fondées sur la recherche. Montréal et Kingston: McGill-Queen's University/Press for the John Deutsch Institute. 


\section{Annexe}

\section{Analyse par régression multivariée classique, échantillon complet filles}

Estimations des paramètres associés avec les variables indépendantes, regroupés par direction (effet positif ou négatif), tous significatifs au seuil de $\left.1 \%{ }^{(* *}\right)$ ou de $5 \%\left({ }^{*}\right)$

\begin{tabular}{ll}
\hline Effet positif ou association positive & Effet négatif ou association négative \\
\hline Profession de la mère & Sens d'appartenance \\
\hline Profession du père & Pression pour réussir \\
\hline Profession future attendue de l'élève & Expérience informatique \\
\hline Joie de lire & École financée en partie par philanthropie \\
\hline Habiletés informatiques & . \\
\hline Maximum du niveau d'éducation des parents & . \\
\hline Avoirs financiers & . \\
\hline Ressources éducatives du ménage & . \\
\hline Temps mis aux devoirs & . \\
\hline Communication culturelle \\
\hline Activités culturelles \\
\hline Lien enseignant-élève \\
\hline Autonomie de l'enseignant ou enseignante & . \\
\hline Manque problématique de personnel d'appui & . \\
\hline Note: Tous sont au seuil $1 \%$.
\end{tabular}

Note: Tous sont au seuil $1 \%$. 


\section{Analyse par régression multivariée classique, échantillon complet garçons}

Estimations des paramètres associés avec les variables indépendantes, regroupés par direction (effet positif ou négatif), tous significatifs au seuil de $1 \%\left({ }^{* *}\right)$ ou de $5 \%\left(^{*}\right)$

\begin{tabular}{|c|c|}
\hline Effet positif ou association positive & Effet négatif ou association négative \\
\hline Profession de la mère* & $\begin{array}{l}\text { Programmes de formation des enseignants et } \\
\text { enseignantes offerts" }\end{array}$ \\
\hline Profession future attendue de l'élève & Manque problématique de personnel d'appui * \\
\hline Joie de lire & $\begin{array}{l}\text { Performance de l'enseignant ou de l'enseignante } \\
\text { évaluée en fonction de la réussite des élèves:" }\end{array}$ \\
\hline Diversité de lecture & Cours pour élèves en difficulté offerts \\
\hline Habiletés informatiques & $\begin{array}{l}\text { Qualité des ressources éducatives de l'école, } \\
\text { d'après l'administration" }\end{array}$ \\
\hline Maximum du niveau d'éducation des parents & Climat disciplinaire de l'école \\
\hline Avoirs financiers: & École financée en partie par philanthropie* \\
\hline Ressources éducatives du ménage & Appui des enseignants et enseignantes \\
\hline Communication culturelle & Expérience informatique \\
\hline Lien enseignant-élève & Sens d'appartenance* \\
\hline Transfert élève pour raison de faible réussite* & . \\
\hline Transfert élève pour raison de besoins spéciaux" & . \\
\hline Matériel arts plastiques insuffisant" & . \\
\hline Cours pour élèves doués offerts" & . \\
\hline $\begin{array}{l}\text { Communication par l'école des résultats scolaires } \\
\text { aux autorités publiques: }\end{array}$ & . \\
\hline Manque problématique de personnel enseignant" & . \\
\hline
\end{tabular}

